\title{
Clinical pharmacology and therapeutics of the oesophagus and the lower oesophageal sphincter
}

\author{
J. J. MisIEWICZ \\ B.SC., M.B., M.R.C.P.E. \\ Medical Research Council Gastroenterology Unit, Central Middlesex Hospital, London
}

\begin{abstract}
Summary
Oesophageal function can be studied with intraluminal manometry, indwelling $\mathrm{pH}$ electrodes or with in vitro methods using isolated tissue. Radiology and endoscopy are the main diagnostic procedures. Innervation of the body of the oesophagus is mainly cholinergic, but the lower oesophageal sphincter appears to be affected by a wide range of biogenic substances, such as autonomic transmitters, polypeptide hormones and prostaglandins. Most drugs used in the treatment of oesophageal disorders act by modifying the action of the naturally occurring agents.
\end{abstract}

\section{Methodology}

Several methods are available for the study of the effects of hormones, drugs and biogenic substances on the human oesophagus, but none are wholly satisfactory. Radiological screening, preferably combined with cinefluorography or video-tape recording, is the single most useful diagnostic technique, but is of limited value to the clinical pharmacologist, because results cannot be quantitated. Similar limitations, compounded by drugs used for sedation and by inflation of the gullet by air, apply to oesophagoscopy. On the other hand the detection of intraluminal pressures by indwelling tubes has provided the main body of data on which the knowledge of oesophageal function is based. A detailed analysis of the sequence of motor events in the gullet can be made from the pressure records. The test is relatively easy to perform, and with care and attention to proper experimental design, adequately controlled quantitative observations of oesophageal and sphincteric responses to various stimuli can be carried out.

The type of pressure sensor placed at the tip of the recording tube must be chosen with care, because it can profoundly influence the configuration and height of the recorded pressure events. Small balloons have been used extensively to collect much valuable data (Fyke, Code and Schlegel, 1956; Atkinson et al., 1957). More recently, Winans and Harris (1967) and Pope (1967) introduced the constantly perfused open-ended tip. This methodological advance has increased the understanding of sphinc- $\frac{\vec{C}}{8}$ teric function and the technique is almost universally 3 . used today. On the debit side, it seems clear that in although steady pressures in the lower oesophageal in sphincter can be recorded for long periods in many subjects, in others a stable record is never obtained. $\vec{\bullet}$ Moreover, sphincteric pressures measured by the $\AA$ pull-through technique vary as each tip passes suc- 을 cessively through the high-pressure zone. The variability of pressures thus recorded may depend on $\frac{7}{O}$ anatomical factors. This may be one reason why the sphincter pressures are of relatively little value in the $\overrightarrow{\vec{v}}$ diagnosis of gastro-oesophageal incompetence (Dil\& wari and Edwards, 1973). Oesophageal manometr however, remains a valid way of studying oese phageal pharmacology and pathophysiology.

It is a truism to say that the clinical consequences of gastro-oesophageal reflux stem from the entry of $\frac{0}{D}$ gastric contents into the oesophagus. This event can $\cong$ be detected by an indwelling pH electrode (Tuttle and $\overrightarrow{\overrightarrow{0}}$ Grossman, 1958; Pattrick, 1970) and the technique 3 can be used to record the frequency of reflux during an extended period, e.g. at night: the effects of drugs and hormones on the occurrence of reflux can also be measured (Stanciu and Bennett, 1972). Leaving aside the controversy that still surrounds the exact mechanism of gastro-oesophageal competence, measurement of intra-oesophageal $\mathrm{pH}$ documents the event that really matters to the patient: whether reflux has occurred or not. It is therefore surprising that this method has not been more widely em- $\rightarrow$ ployed (Bennett, 1973). It is worth mentioning that results of reflux tests performed after intragastric N instillation of acid may be affected to some extent by changes in the level of circulating gastrin produced by low antral $\mathrm{pH}$ and consequent alterations in cardiac sphincter pressures.

Recording of electrical potential swings in vivo is possible in man, and exploration of this variable $ळ$ may yield interesting data in the future. In vitro pharmacological studies of strips of oesophageal muscle from surgical resection specimens allow an analysis to be made of the innervation of oesophageal muscle and its responses to drugs, hormones 


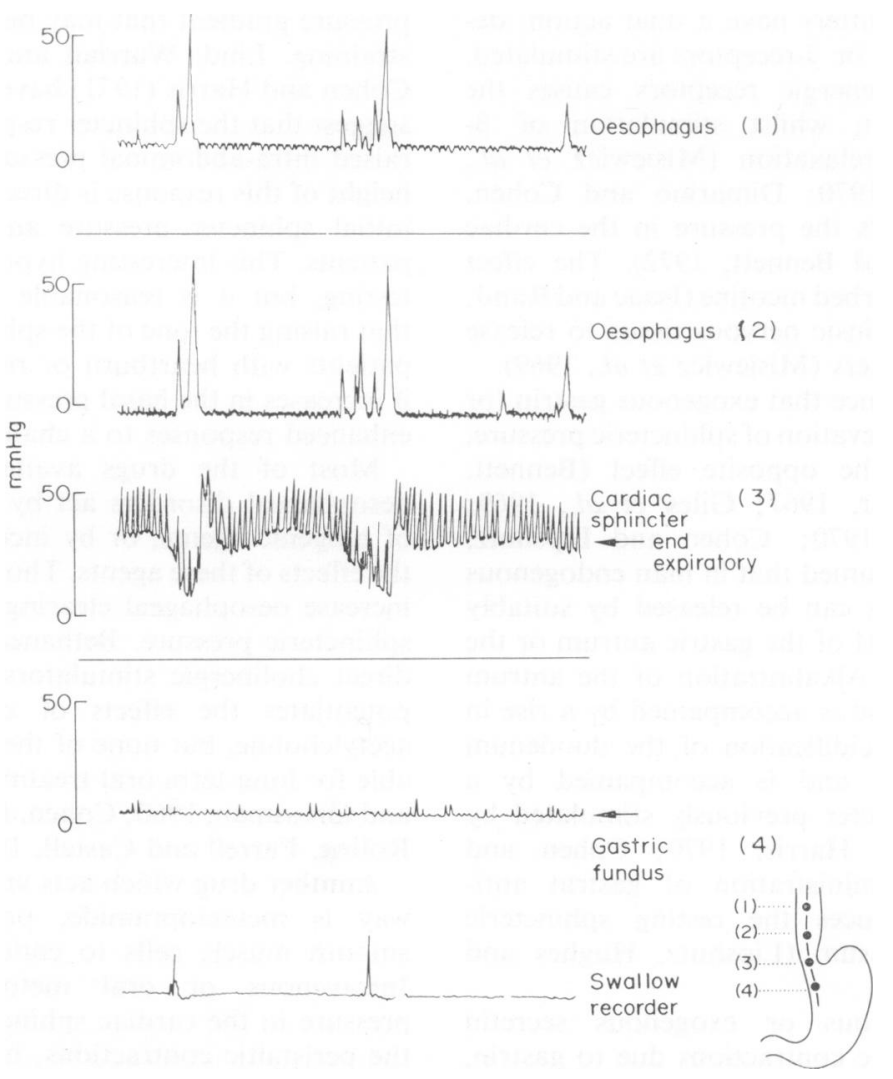

FIG. 1. Intraluminal pressures recorded with perfused open-ended tips from the body of the oesophagus (tubes 1 and 2), the lower oesophageal sphincter (tube 3) and the fundus (tube 4).

or electrical stimulation in normal and pathological states. This method allows a far more detailed and precise analysis of these aspects of oesophageal function than is possible in vivo, but the limitations of an in vitro method should be borne in mind when extrapolating the results to intact man.

\section{Pharmacology and therapeutics}

The sequence of pressure events in the normal oesophagus is illustrated in Fig. 1. The lower oesophageal sphincter exerts a resting pressure of some $10-40 \mathrm{mmHg}$; the pressure in the body of the oesophagus is sub-atmospheric. In response to swallowing, an orderly peristaltic contraction sweeps distally down the oesophagus at $2-4 \mathrm{~cm} / \mathrm{sec}$. The lower oesophageal sphincter relaxes rapidly, the pressure falling to basal (gastric fundal) level. The sphincter is fully relaxed by the time the peristaltic wave reaches it; and following an after-contraction, the pressure returns to its original level.

Peristaltic activity in the body of the oesophagus is probably mediated through a cholinergic pathway.
In vitro experiments show the intrinsic nerves of the oesophagus to be predominantly cholinergic and the contractions are inhibited by atropine (Adams et al., 1961; Kantrowitz, Siegel and Hendrix, 1966). Pentagastrin has no effect on the motility of the oesophageal body, indicating that receptors for polypeptide hormones are absent in that part of the gullet (Hollis, Levine and Castell, 1972).

Control of the lower oesophageal sphincter appears to be more complex, because it is affected not only by neurotransmitters, but also by polypeptide hormones and by other biogenic substances. It may be convenient to divide the motor events in the cardiac sphincter into two categories: (a) a fast component of relaxation and contraction during swallowing, which is probably mediated through nerves, and (b) a slow component-the steady sphincteric pressure, which is affected by circulating polypeptide hormones or locally released mediators.

The classical parasympathetic and sympathetic mediators affect the cardiac sphincter in different ways. Acetylcholine increases sphincteric pressure, 
but adrenergic transmitters have a dual action, depending on whether $\alpha$ - or $\beta$-receptors are stimulated. Stimulation of $\alpha$-adrenergic receptors causes the sphincter to contract, whilst stimulation of $\beta$ receptors results in relaxation (Misiewicz et al., 1969; Zfass et al., 1970; Dimarino and Cohen, 1973). Smoking lowers the pressure in the cardiac sphincter (Stanciu and Bennett, 1972). The effect may be due to the absorbed nicotine (Isaac and Rand, 1972) causing the intrinsic nervous tissue to release $\beta$-adrenergic transmitters (Misiewicz et al., 1969).

There is good evidence that exogenous gastrin (or pentagastrin) causes elevation of sphincteric pressure, whilst secretin has the opposite effect (Bennett, Misiewicz and Waller, 1967; Giles et al., 1969; Castell and Harris, 1970; Cohen and Lipshutz, 1971). It is usually assumed that in man endogenous polypeptide hormones can be released by suitably altering the luminal $\mathrm{pH}$ of the gastric antrum or the proximal duodenum. Alkalinization of the antrum may release gastrin and is accompanied by a rise in sphincter pressure; acidification of the duodenum may release secretin, and is accompanied by a relaxation of a sphincter previously stimulated by gastrin (Castell and Harris, 1970; Cohen and Lipshutz, 1971). Administration of gastrin antiserum markedly reduces the resting sphincteric pressure in the opossum (Lipshutz, Hughes and Cohen, 1972).

Although endogenous or exogenous secretin antagonizes sphincteric contractions due to gastrin, the tone of the resting sphincter is not affected, suggesting that substances other than gastrin may maintain basal sphincter tone. This hypothesis is strengthened by the observations of Dilawari et al. (1973), who reported that exogenous prostaglandin $\mathrm{F}_{2} \alpha$ increased sphincter pressure, whilst prostaglandin $E_{2}$ diminished the effect of pentagastrin. Inhibition of prostaglandin synthetase by indomethacin resulted in increased sphincter pressure, indicating a suppression of an endogenous inhibitory prostaglandin-possibly prostaglandin $\mathrm{E}_{2}$. Yet other pathways-such as the non-adrenergic inhibitory pathway-may be also concerned in the control of sphincter tone.

These data have certain therapeutic implications. In general, pressures in the cardiac sphincter tend to be lower in patients with gastro-oesophageal reflux than in controls (Winans and Harris, 1967), although the overlap between the two groups is considerable in most reported series and the pressure has to be very low $(<6.5 \mathrm{mmHg}$ ) for reflux to be diagnosable manometrically (Pope, 1967; Dilawari and Edwards, 1973). The magnitude of normal sphincteric squeeze $(10-40 \mathrm{mmHg}$ ) makes it difficult to accept that it of itself constitutes a barrier capable of withstanding the sometimes considerable gastro-oesophageal pressure gradient that may be generated, e.g. during straining. Lind, Warrian and Wanklin (1966) and Cohen and Harris (1971) have published data which suggest that the sphincter responds to a challenge of raised intra-abdominal pressure by contracting: the height of this response is directly proportional to the initial sphincter pressure and is less in refluxing patients. This interesting hypothesis requires further testing, but it is reasonable to assume meanwhile that raising the tone of the sphincter is worthwhile in patients with heartburn or regurgitation, especially if increases in the basal pressure are accompanied by enhanced responses to a challenge.

Most of the drugs available for treatment of oesophageal disorders act by modifying the release of biogenic agents, or by increasing or diminishing the effects of these agents. Thus cholinergic drugs will increase oesophageal clearing of acid and raise the sphincteric pressure. Bethanechol or urecholine are direct cholinergic stimulators, whilst edrophonium potentiates the effects of endogenously released acetylcholine, but none of these substances are suitable for long-term oral treatment (Bertanello, Tuttle and Grossman, 1960; Cohen, Fisher and Tuch, 1972; Roling, Farrell and Castell, 1972).

Another drug which acts via the cholinergic pathe way is metoclopramide, possibly by sensitizing smooth muscle cells to endogenous acetylcholine Intravenous or oral metoclopramide increases pressure in the cardiac sphincter and amplitudes of the peristaltic contractions, but the response of the cardiac sphincter is greatest in those with highest basal pressures, which may set a limit to the effectiveness of the drug (Heitman and Möller, 1970; Dilawari and Misiewicz, 1972). The effects of metoclopramide are interesting pharmacologically, but its superiority to conventional treatment of heartburn with antacids is yet to be shown in formal therapeutic trials.

It follows from the pharmacological data summarized above, that anticholinergic preparations are likely to produce unwanted effects on the oesophagus and its lower sphincter. For example, atropine diminishes both the sphincteric pressure and the amplitude of peristaltic contractions and is said to be followed by gastro-oesophageal reflux (Kantrowitz et al., 1966; Betarello et al., 1960). Anticholinergic drugs should therefore not be used in the treatment of heartburn or regurgitation.

The work on polypeptide hormones suggests that antacids may act not only by neutralizing the acid, 0 but also by promoting the release of antral gastrin $\varnothing$ with the consequent rise in the tone of the cardiac $\stackrel{\mathcal{P}}{+}$ sphincter rendering the oesophago-gastric junction 0 competent. This action would depend on making the antrum alkaline and there is no information available at the moment whether any antacid is 
better for this purpose than another. The role of prostaglandin analogues or antagonists in the treatment of abnormal oesophageal motility belongs to future research.

\section{Acknowledgment}

Figure 1 is reproduced from Gut (1973) 14, 244 by kind permission of the Editor.

\section{References}

Adams, C.W.M., Brain, R.F.H., Ellis, F.G., Kauntze, R. \& Trounce, J.R. (1961) Achalasia of the cardia. Guy's Hospital Reports, 110, 191.

Atkinson, M., Edwards, D.A.W., Honour, A.J. \& RowLANDS, E.N. (1957) Comparison of cardiac and pyloric sphincters: a manometric study. Lancet, ii, 918.

BenNeTt, J.R. (1973) Symposium on gastro-oesophageal reflux and its complications: Section 5. The physician's problem. Gut, 14, 246.

Bennett, A., Misiewicz, J.J. \& Waller, Sheila L. (1967) Analysis of the motor effects of gastrin and pentagastrin on the human alimentary tract in vitro. Gut, 8, 407.

Betarello, A., Tuttle, S.G. \& Grossman, M.I. (1960) Effect of autonomic drugs of gastro-esophageal reflux. Gastroenterology, 39, 240.

Castell, D.O. \& HarRis, L.D. (1970) Hormonal control of gastro-esophageal sphincter-strength. New England Journal of Medicine, 282, 886.

Cohen, S., Fisher, R. \& Tuch, A. (1972) The site of denervation in achalasia. Gut, 13, 556.

Cohen, S. \& Harris, L.D. (1971) Does hiatus hernia affect competence of the gastro-esophageal sphincter? New England Journal of Medicine, 284, 1053.

Cohen, S. \& Lipshutz, W. (1971) Hormonal regulation of human lower esophageal sphincter competence: interaction of gastrin and secretin. Journal of Clinical Investigation, 50, 449.

Dilawari, J.B. \& Edwards, D.A.W. (1973) Cardiac sphincter pressure and the probability of radiographic evidence or symptoms of reflux. (Abstract.) Rendiconti di Gastro-enterologia, 5, 27.

Dilawari, J.B. \& Misiewicz, J.J. (1973) Action of oral metoclopramide on the gastro-oesophageal junction in man. Gut, 14, 380.

Dilawari, J.B., Newman, A., Poleo, J. \& Misiewicz, J.J. (1973) The effect of prostaglandins and of anti-inflammatory drugs on the oesophagus and the cardiac sphincter in man. (Abstract.) Gut, 14, 822.

Dimarino, A.J. \& Cohen, S. (1973) The adrenergic regulation of lower esophageal sphincter function: a model of denervation supersensitivity. Clinical Research, 20, 869.
FyKe, F.E., Code, C.F. \& Schlegel, J.F. (1956) The gastroesophageal sphincter in healthy human beings. Gastroenterology, 86, 135.

Giles, G.R., Mason, M.C., Humphries, C. \& Clark, C.G. (1969) Action of gastrin on the lower oesophageal sphincter in man. Gut, 10, 730 .

HeitmanN, P. \& Möller, N. (1970) The effect of metoclopramide on the gastro-esophageal junctional zone and the distal esophagus in man. Scandinavian Journal of Gastroenterology, 5, 621 .

Hollis, J.B., Levine, S.M. \& Castell, D.O. (1972) Differential sensitivity of the human esophagus to pentagastrin. American Journal of Physiology, 222, 870.

IsAAC, P.F. \& RAND, M.J. (1972) Cigarette smoking and plasma levels of nicotine. Nature, 236, 308.

Kantrowitz, P.A., Siegel, C.I. \& Hendrix, T.R. (1966) Differences in motility of the upper and lower esophagus in man and its alteration by atropine. Bulletin Johns Hopkins Hospital, 118, 476.

Lind, J.F., WARRIAN, W.G. \& WANKLin, W.J. (1966) Responses of the gastro-esophageal junctional zone to increases in abdominal pressure. Canadian Journal of Surgery, 9, 32.

Lipshutz, W., Hughes, W. \& Cohen, S. (1972) The genesis of lower esophageal sphincter pressure: its identification through the use of gastrin antiserum. Journal of Clinical Investigation, 51, 522 .

Misiewicz, J.J., Waller, Sheila L., Anthony, P.P. \& Gummer, J.W.P. (1969) Achalasia of the cardia: pharmacology and histopathology of isolated cardiac sphincteric muscle from patients with or without achalasia. Quarterly Journal of Medicine, 38, 17.

PAtTrick, F.G. (1970) Investigation of gastro-oesophageal reflux in various positions with a two-lumen $\mathrm{pH}$ electrode. Gut, 11, 659.

Pope, C.E. (1967) A dynamic test of sphincter strength: its application to the lower esophageal sphincter. Gastroenterology, 52, 779.

Roling, G.T., Farrell, R.L. \& Castell, D.O. (1972) Cholinergic response of the lower esophageal sphincter. American Journal of Physiology, 222, 967.

Stanciu, C. \& BenNetT, J.R. (1972) Smoking and gastrooesophageal reflux. British Medical Journal, 3, 793.

Tuttle, S.G. \& Grossman, M.I. (1958) Detection of gastroesophageal reflux by simultaneous measurement of intraluminal pressure and $\mathrm{pH}$. Proceedings of the Society of Experimental Biology (N.Y.), 98, 225.

WinANS, C.S. \& HARRIS, L.D. (1967) Quantitation of lower esophageal sphincter competence. Gastroenterology, 52, 773.

Zfass, A.M., Prince, R., Allen, F.N. \& Farrar, J.J. (1970) Inhibitory $\beta$-adrenergic receptors in the human distal esophagus. American Journal of Digestive Diseases, 15, 303 . 